\title{
The Combination of Red Yeast Rice Extract, Oatmeal and Olive Oil Reduces Serum Cholesterol
}

\author{
Amila Oshan Silva, MD, PhD* \\ Department of Clinical Science, Kaiser Permanente Bernard J Tyson School of Medicine, USA
}

\begin{abstract}
Elevated serum cholesterol is a significant risk factor for morbidity and mortality. The main approach to reduce cholesterol is the use of statin drugs. The current study tested the use of a novel food-based technique to reduce cholesterol - the combination of red yeast rice extract (RYRE), extra virgin olive oil (EVOO) and oatmeal. The study was a non-randomized prospective intervention that enrolled 86 patients with elevated total and low-density lipoprotein cholesterol (LDL-C). It assessed whether daily consumption of above components would reduce total cholesterol and LDL-C. Subjects were instructed to consume either $2400 \mathrm{mg}$ or $3600 \mathrm{mg}$ of RYRE daily based on their initial serum cholesterol value and asked to use EVOO for cooking and eat 1.5 cups of oatmeal daily. When fasting lipid panels were repeated at 3 and 6 months $90 \%$ of females and $80 \%$ of males demonstrated a clinically significant reduction of total cholesterol and LDL-C. Among a subgroup that was more compliant, total cholesterol was reduced by $22 \%$ and LDL-C was reduced by $31.5 \%$. There was no change in HDL-Cholesterol. The study demonstrates that two commonly consumed foods, oatmeal and EVOO, and a widely available over the counter supplement, RYRE, can reduce serum cholesterol levels similar to the effect of statins.
\end{abstract}

\section{Keywords}

Extra virgin olive oil, High density lipoprotein cholesterol, Low density lipoprotein cholesterol, Oatmeal, Red yeast rice extract, Total Cholesterol

\section{Introduction}

Elevated cholesterol is a prevalent risk factor that causes morbidity and mortality. Despite the awareness of high cholesterol as a major health concern, nearly 100 million Americans have elevated cholesterol, and of those, less than $30 \%$ have their cholesterol levels under control [1]. Medical conditions associated with hypercholesterolemia include heart disease and stroke, the two leading causes of death in this country. Studies have also shown a positive correlation between hypercholesterolemia and obesity, the latter being another major health problem in the USA.

In addition to serious health consequences, hypercholesterolemia is a significant economic burden. The estimated total cost of treatment of cardiovascular diseases in the United States, many of which are related to hypercholesterolemia, exceeds 400 billion dollars annually. This accounts for nearly $15 \%$ of all national healthcare costs. Thus, it represents an enormous economic burden and underscores the need to treat hypercholesterolemia in a timely and cost-effective manner.

The most common treatment modality for patients with hypercholesterolemia is medical management with the cholesterol-lowering statin drugs [2]. Statins antagonize the synthesis of cholesterol in multiple tissues and increases the production of the receptors on liver cells that clear lowdensity lipoprotein cholesterol (LDL-C) from circulation [3]. The treatment cost of an individual with statins remains quite high even with the availability of generic drugs. The annual cost of treatment with a generic drug is $\$ 313$ and the number increases substantially to up to $\$ 14,000$ for non-generics [4].

An alternative approach to lowering cholesterol levels is to initiate a change in lifestyle, such as a change in eating and exercise habits. Many foods and supplements have been promoted as "health foods" that help lower cholesterol. These include garlic, avocadoes, spinach, canola oil, extra virgin olive oil (EVOO) and oatmeal to name a few [5]. Some claims about these popular health foods have not stood the test of scientific scrutiny. For instance, a large randomized

*Corresponding author: Amila Oshan Silva, MD, PhD, Department of Clinical Science, Kaiser Permanente Bernard J Tyson School of Medicine, $100 \mathrm{~S}$. Los Robles Avenue, Pasadena CA 91101, USA

Accepted: July 20, 2021

Published online: July 22, 2021

Citation: Silva AO (2021) The Combination of Red Yeast Rice Extract, Oatmeal and Olive Oil Reduces Serum Cholesterol. J Hum Nutr 4(1):130-135 
controlled trial that enrolled 192 patients to examine the effectiveness of garlic found no benefit in lowering LDL-C or total cholesterol [6]. Similarly, there is very limited evidence to support that canola oil or spinach lowers serum cholesterol levels significantly $[7,8]$.

Two foods that have been shown in clinical studies to lower cholesterol are oatmeal and EVOO. Oat $\beta$-glucan, a soluble dietary fiber that is found in the endosperm of oats has been shown to lower cholesterol [9]. Studies have shown that intake of oat $\beta$-glucan at daily doses of $3 \mathrm{~g}$ may reduce total cholesterol and LDL-C levels by $5-10 \%[9,10]$. As a result, oat beta-glucan is one of the few dietary components that have been approved by the FDA to have a claim that states "As part of a healthy diet, the soluble fiber in oatmeal helps remove cholesterol". EVOO has also been shown to lower both total cholesterol and LDL-C [11,12]. EVOO is high in monounsaturated fats, a source of bioactive phenolic compounds and a key element of the Mediterranean diet [11]. It has been shown that dietary supplementation with EVOO reduced total cholesterol and LDL-C [12]. A diet supplemented with EVOO has been shown to promote primary prevention of cardiovascular disease [12].

A dietary supplement that has shown efficacy in reducing cholesterol levels is red yeast rice extract (RYRE). There is documentation of its use in China dating back to $800 \mathrm{AD}$ and it is widely consumed in other Asian countries such as Japan and Korea [13]. It is manufactured by culturing Monascuspurpureus yeast on white rice at carefully controlled growing conditions to increase the concentrations of chemicals that lower blood cholesterol and triglycerides [13]. The primary cholesterol-lowering bioactive constituent in RYRE is monacolin K, which has a similar chemical structure to the cholesterol lowering statin medication, lovastatin [14]. Similar to lovastatin, monacolin $\mathrm{K}$ and other substances found in RYRE inhibit the rate-limiting enzyme in cholesterol biosynthesis, 3-hydroxy-3-methylglutaryl coenzyme A (HMG-CoA) reductase. In addition to monacolin $\mathrm{K}$, RYRE has been found to contain sterols ( $\beta$-sitosterol, campesterol, stigmasterol, and sapogenin), isoflavones and isoflavone glycosides, and monounsaturated fatty acids, which may also contribute to reducing cholesterol levels. These other substances likely contribute to reducing cholesterol levels since the quantities of the HMG-CoA reductase inhibitory constituents contained in RYRE are inadequate to explain the magnitude of its cholesterol-lowering effects relative to statins [14].

Several studies have shown that RYRE is effective in lowering cholesterol. Studies conducted in China have shown dietary supplementation with RYRE to reduce cholesterol by $11-32 \%$ [15]. In the US, studies have shown total cholesterol by reduction of $16 \%$ to $18 \%$ with dietary supplementation of RYRE $[14,16]$.

While RYRE, oatmeal and EVOO each individually lower cholesterol, they have not been as effective as statins. Less potent statins typically reduce cholesterol by $20 \%$ and more potent can reduce cholesterol by up to $50 \%[17,18]$. This study assessed whether the combination of RYRE, oatmeal and EVOO could be as effective as a statin to reduce total cholesterol and LDL-C. If effective, this dietary approach can potentially replace statins as the first line treatment for lowering plasma cholesterol levels.

\section{Materials and Methods}

Study design: Non-randomized prospective intervention.

The study design and procedures were approved by the Kaiser Permanente IRB. The written informed consent was reviewed and signed by each subject before enrollment.

The inclusion criteria for participation were as follows: Male and female patients aged 40-70 years with total cholesterol ranging from $200-270 \mathrm{mg} / \mathrm{dL}$ and LDL-C ranging from $115-200 \mathrm{mg} / \mathrm{dL}$. Prior to study enrollment, all patients underwent assessment for eligibility via performance of a screening fasting lipid profile. The following patients were excluded from study enrollment: 1) Those treated previously for hypercholesterolemia and had abnormal liver function test results; 2) Those with a history of statin-associated myositis or rhabdomyolysis, myocardial infarction, stent, or coronary artery bypass grafting; 3 ) Those taking any lipidregulating drugs, hormone replacement therapy, drugs known to affect lipid concentrations, or supplements known to affect lipid levels; 4) Those with an endocrine disease known to lead to lipid abnormalities; and 5) Those who selfreported pregnancy, lactation, current smoking, prevalent heart disease, or cancer.

Study patients were instructed to consume either 2400 $\mathrm{mg}(4 \times 600 \mathrm{mg} /$ capsule $)$ or $3600 \mathrm{mg}(6 \times 600 \mathrm{mg} /$ capsule $)$ of RYRE (Jarrow Formulas, Los Angeles, CA, USA) daily, only use EVOO for cooking (maximum 2 tablespoons/day) and eat 1.5 cups of oatmeal (Bob's Red Mill, Milwaukie, OR, USA) daily. Patients whose total cholesterol was 200 to $225 \mathrm{mg} /$ dl were instructed to consume $2400 \mathrm{mg}$ of RYRE, whereas patients whose total cholesterol was greater than $225 \mathrm{mg}$ were instructed to consume $3600 \mathrm{mg}$ of RYRE.

Subjects were provided a food diary with instructions to complete it to monitor their intake of the dietary interventions. Subjects also had an end of study interview to assess compliance to the study protocol.

Fasting lipid panels, complete blood count (CBC), liver function tests and chemistry panels were measured at 0,3 and 6 months. The food diary was analyzed to assess patient compliance level.

In terms of statistical analysis, the study was powered to detect a $12 \%$ reduction in LDL-C with an anticipated $19 \mathrm{mg} /$ $\mathrm{dL}$ standard deviation for change in LDL-C levels after the initiation of the intervention. For a target type I error rate of 0.05 and standard deviation for LDL-C $=15$, we estimated at least 80 subjects were needed to have a power of $80 \%$. Considering an anticipated $5 \%$ dropout rate, 86 patients were recruited to the study.

\section{Results}

120 patients were screened, and 86 patients enrolled in the study ( 37 men and 49 women). The patients represented 
a variety of racial backgrounds, indicative of the ethnic diversity in the area where the study was conducted. Twelve subjects did not complete the first set of follow-up labs. The most common reason for subject withdrawal was due to scheduling conflicts. Other reasons included a development of an unrelated medical condition, difficulty swallowing pills, being started on a lipid lowering medication by their primary care physician or change in health insurance plans. Only one patient withdrew due to a side-effect from the study regimen. The patient had increased bowel gas after initiating the study regimen.

\section{Baseline demographics and Protocol Compliance rates}

74 patient completed 3 months of the study and 68 remained enrolled for the entire duration of the study. Baseline demographics of the patients who completed 3 months of the study are shown in Table 1.

Compliance rates were assessed by examination of the food diary and an exit inter-view. In the exit interview, patients were asked how many days in each week they complied with the study protocol ranging from 1 through 7 days. A subgroup analysis was performed based on compliance rates. Patients who complied with the protocol at least 6 days of the week were considered highly compliant. 36 of the 68 patients who completed the six months of the study were deemed to be highly compliant per the criteria.

\section{Effect of study protocol on Serum Cholesterol levels}

At 3 months, the average reduction from baseline of total cholesterol and LDL-C was $15.2 \%$ and $22.4 \%$, respectively. At 6 months, the average reduction from baseline of total cholesterol and LDL-C was $15.8 \%$ and $21.83 \%$, respectively. There was no statistically sig-nificant change in HDL-C cholesterol, triglycerides or ALT levels at either time point. At 3 months only 3 of the participants did not have a decline in total cholesterol (4\%) and at 6 months only 1 patient did not have a decline in comparison in the initial values (1.5\%).

A sample t-test was conducted to examine if the percent change in LDL-C was different from -24\% (alpha $=0.05,2$-sided

Table 1: Baseline Demographics of Patients who completed first 3 months of study.

\begin{tabular}{|l|l|}
\hline Age (yrs) & 56.2 \\
\hline Mean & 58.0 \\
\hline Median & $48.0,64.0$ \\
\hline Q1, Q3 & \\
\hline Gender & $42(56.8 \%)$ \\
\hline Female & $32(43.2 \%)$ \\
\hline Male & \\
\hline Ethnicity & $11(14.9 \%)$ \\
\hline Asian/Pacific Islander & $8(10.8 \%)$ \\
\hline Black & $31(41.9 \%)$ \\
\hline Hispanic & $18(24.3 \%)$ \\
\hline White & $6(8.1 \%)$ \\
\hline Other/Unknown & \\
\hline
\end{tabular}

test, Figure 1). The $24 \%$ value was assigned since that is the average reduction of LDL-C observed in patients prescribed low dose statins [17]. The percent reduction of LDL-C after 6 months was $-21.83 \%$ (95\% CL $-24.66 \%,-19.00 \%$ ), which was not statistically significantly different from $-24 \%$ achieved by statins after 6 months of treatment $(p=0.13)$. Thus, the T-test procedure demonstrates equivalency when comparing the reduction of LDL-C between low dose statins and the study regimen.

Among highly compliant patients, at 6 months, the average reduction from baseline of LDL-C and total cholesterol was $30 \%$ (Figure 2) and $21.4 \%$, respectively. There was no significant change in HDL-C, triglycerides or ALT levels in this subgroup either. The median percent reduction of LDL-C in this subset of patients after 6 months was $-27.40 \%$ (95\% CL $-30.6 \%,-25.2 \%)$, which was statistically significantly lower than $-24 \%$ by statin in 6 -month treatment $(p<0.001)$.

\section{Discussion}

The present interventional prospective study assessed whether daily ingestion of RYRE, EVOO and oatmeal could reduce total cholesterol and LDL-C by a clinically significant extent. Among all participants, this dietary intervention lowered total cholesterol and LDL-C similar to the levels achieved by low dose statins, a prescription medication. Furthermore, there was no significant change in the HDL-C cholesterol. This is a significant development in the treatment of hypercholesterolemia. Patients with elevated cholesterol would have a clinically proven over the counter alternative to lower cholesterol that is as effective as a low dose statin.

Each ingredient used in this study is relatively safe. Oatmeal has been a staple of Western diets for centuries. It is made using hulled oat grains and is recommended as a breakfast cereal for individuals older than 2 years of age. Oat flour is also used to make numerous foods that are consumed worldwide [19]. EVOO is derived from the fruit of the olive and is a traditional oil in the Mediterranean region. Its use heralds back to 8th century $B C$ and there are no known side effects of consuming olive oil [20]. The use of red yeast rice (RYR) in China was first documented in the Tang Dynasty in 800 AD [13]. It has been used to make rice wine, as a food preservative for maintaining the color and taste of fish and meat, and for medicinal properties. Medieval Chinese medical literature mentions RYR as a substance that "improves circulation". RYR continues to be a dietary staple in many Asian countries, including China and Japan, with daily consumption up to 50 grams [13].

The mechanism by which each ingredient lowers cholesterol is unique. Oatmeal reduces cholesterol via a component known as beta-glucan which blocks the absorption of bile acids and cholesterol in the intestine and promotes their excretion. Beta-glucan may also lower cholesterol by undergoing bacterial fermentation and releasing short-chain fatty acids which may reduce cholesterol synthesis through inhibition of the enzyme HMG-CoA reductase [4]. EVOO is postulated to reduce cholesterol through the promotion of LDL-C efflux from the body. Phenolic components of EVOO reduces oxidative modifications to HDL-C and by 


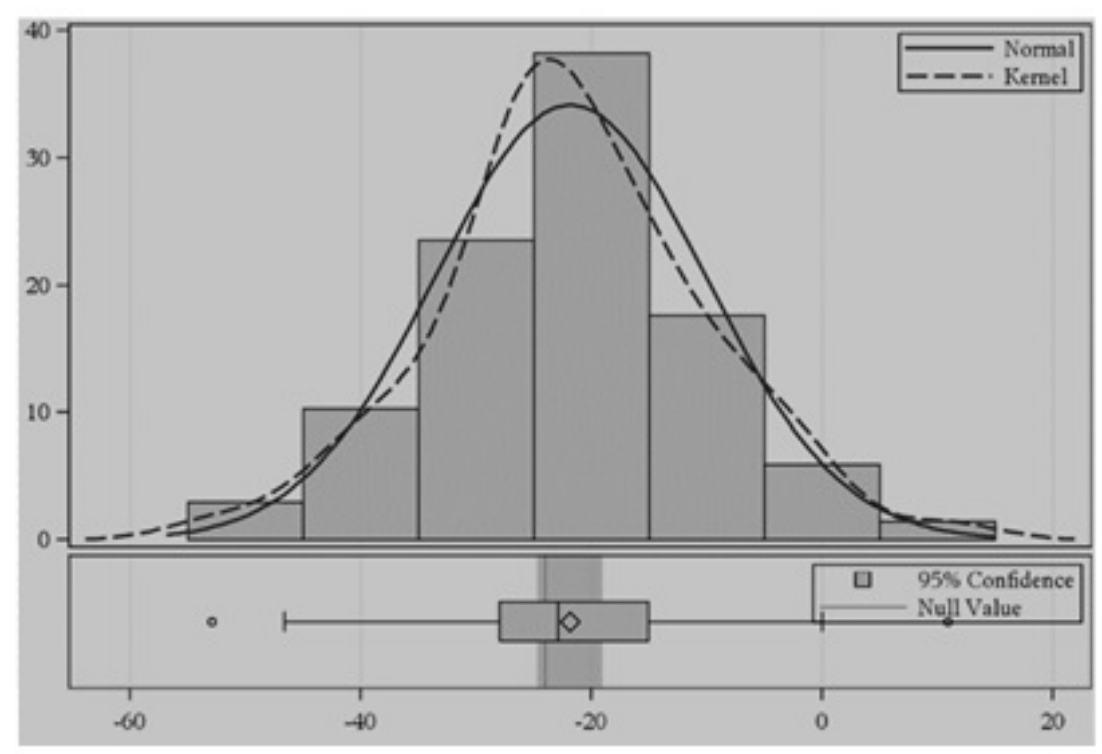

Figure 1: T- Test Procedure demonstrating Equivalency in LDL reduction of study regimen and statins at 6 months (with $95 \%$ Confidence interval for mean).

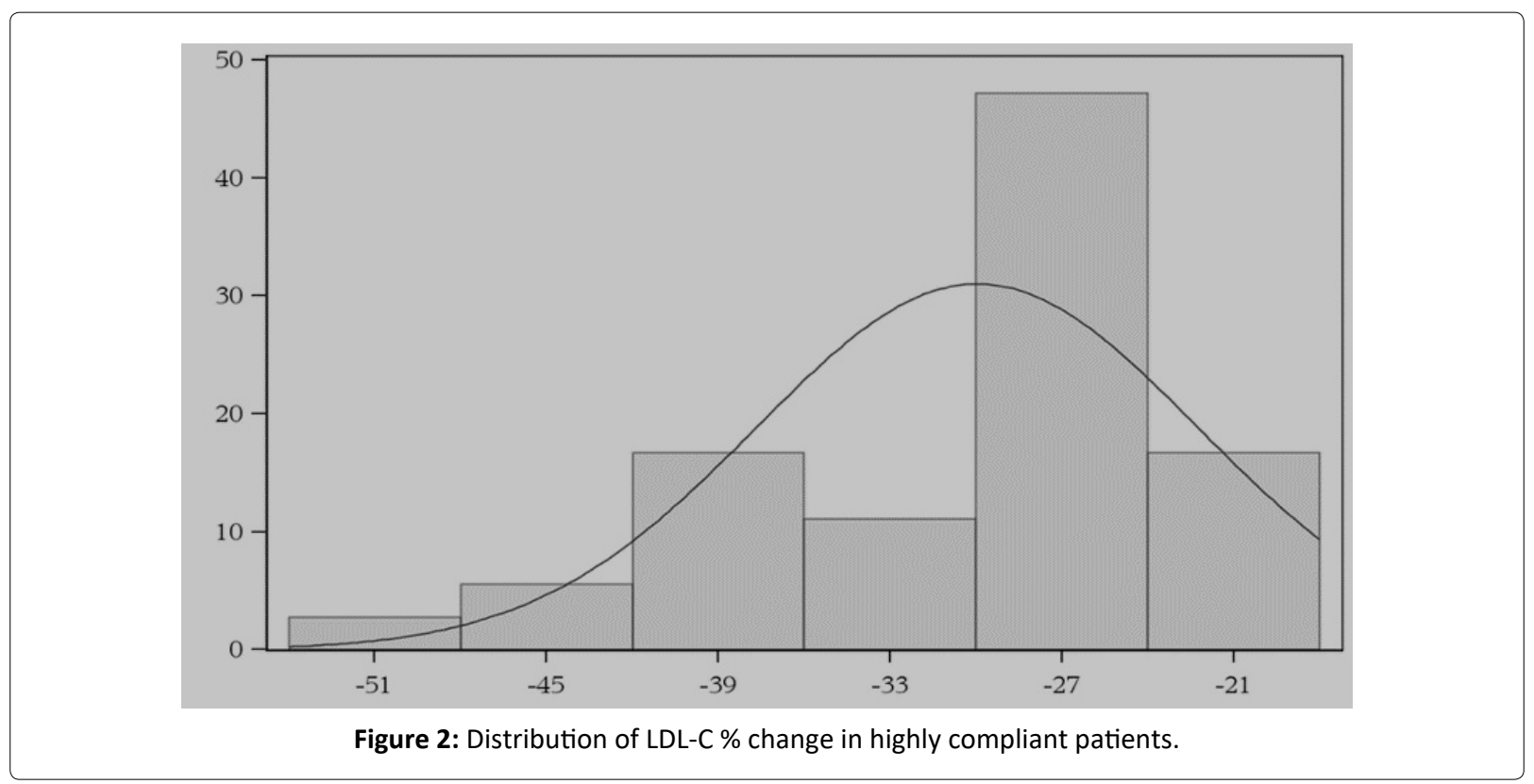

maintaining the physicochemical properties of $\mathrm{HDL}-\mathrm{C}$, improves the functionality of HDL-C, especially the capacity to promote cholesterol efflux [15]. RYRE has been known to contain substances that lower cholesterol [13]. These include monacolins, which are like statins and other substances such as sterols and isoflavones.

While oatmeal, EVOO and RYRE have each been shown to lower cholesterol, the level of LDL-C reduction achieved by the combination of the three ingredients is quite impressive. Based on past studies, one would expect the reduction to be around $20 \%$. However, highly compliant study participants achieved a reduction of around $30 \%$. This level of reduction indicates the three ingredients may be working in a synergistic manner to reduce cholesterol.

There was varied response to the study protocol. For instance, a subset of study patients, who were identified as highly compliant in the exit interview, had a significantly greater reduction in cholesterol levels in comparison to the remainder of the group. This group's LDL cholesterol reduction was $30 \%$, greater than the reduction achieved by low dose statins. Among the remainder of the study subjects, cholesterol reduced to a lesser extent and in one patient there was no reduction in cholesterol levels. One factor that likely affected the efficacy of the study protocol was the level of patient compliance. On the exit survey, the main factor that reduced compliance was the requirement to eat oatmeal daily. Patients stated that after a few weeks they became "tired" of eating oatmeal and desired other breakfast options. Patients also stated that it would be convenient if the components used in the study were available in a pill form. Inspired by patient feedback, additional research was undertaken, and a single pill has been developed that contains all three components of the study protocol. 
Another factor that almost certainly affected patient response was individual variation in diet. This study did not require that patient adhere to any particular diet. Patients were given commonly known dietary information in a handout form that comprised of healthy eating guidelines recommended by the U.S. Department of Agriculture. The only dietary stipulations were exclusive use of olive oil as the form of oil in food preparation and daily consumption of oatmeal. The rationale for this approach was to assess the effectiveness of the study regimen in a community setting where patients could easily incorporate the study protocol into their daily routine. Multiple studies have shown dramatic changes in existing diets are rarely sustained long-term $[21,22]$.

This study provides a novel, straightforward approach to effectively reduce LDL-C without utilizing prescription medications. The combination of RYRE, EVOO and oatmeal offers a new way to reduce cholesterol by an extent that is usually achieved by prescription medications. Such an approach to reduce cholesterol would translate into significant cost savings for the medical system, since an over the counter approach will not have associated physician visit and pharmacy costs. Most importantly, this combination of ingredients to lower cholesterol will have broad appeal to a generation of individuals seeking natural, nutritional approaches to attain good health.

\section{Funding}

This research was funded by Regional Research Committee, Southern California Permanente Medical Group Department of Research \& Evaluation 11980 Telegraph Rd, Suite 200, Santa Fe Springs, CA 90670. RRC Grant No: KP-RRCYEAR20160408.

\section{Data Availability Statement}

The data utilized in the study does include confidential patient data and has been stored securely. All data is available for review and requests will be honored in accordance with the data sharing policies of the Kaiser Permanente IRB.

\section{Acknowledgments}

I would like to thank Kaiser Permanente physicians at the Ontario Medical Center who helped enroll patients for the study. I would also like to thank Dr. Anthony Thomas (Natreon Inc, NJ) who helped review the draft manuscript and provided valuable advice for the study design. I am grateful to Qiaowu (Zoe) Li, MS for her expert analysis of the data and for de-signing the figures used in the publication. I would like to acknowledge Jarrow Formulas for the donation of RYRE Capsules and Bob's Red Mill Corporation for the donation of oatmeal.

\section{References}

1. Mozaffarian D, Benjamin EJ, Go AS, et al. (2015) Heart disease and stroke statistics-2015 update: A report from the American heart association. Circulation 131: e29-e322.

2. Gotto AM Jr (2002) Statins: Powerful drugs for lowering cholesterol. Circulation 105: 1514-1516.
3. Ahmadi Y, Ghorbanihaghjo A, Argani H (2017) The effect of statins on the organs: Similar or contradictory? J Cardiovasc Thorac Res 9: 64-70.

4. Jick H, Wilson A, Wiggins $P$, et al. (2012) Comparison of prescription drug costs in the United States and the United Kingdom, Part 1: Statins. Pharmacotherapy 32: 1-6.

5. Eilat-Adar S, Sinai T, Yosefy C, et al. (2013) Nutritional recommendations for cardiovascular disease prevention. Nutrients 5: 3646-3683.

6. Gardner CD, Lawson LD, Block E, et al. (2007) Effect of raw garlic vs. commercial garlic supplements on plasma lipid concentrations in adults with moderate hypercholesterolemia: A randomized clinical trial. Arch Intern Med 167: 346-353.

7. Ghobadi S, Hassanzadeh-Rostami Z, Mohammadian F, et al. (2019) Effects of canola oil consumption on lipid profile: A systematic review and meta-analysis of randomized controlled clinical trials. J Am Coll Nutr 38: 185-196.

8. Rebello CJ, Chu J, Beyl R, et al. (2015) Acute effects of a spinach ex-tract rich in thylakoids on satiety: A randomized controlled crossover trial. J Am Coll Nutr 34: 470-477.

9. Othman RA, Moghadasian MH, Jones PJ (2011) Cholesterollowering effects of oat $\beta$-glucan. Nutr Rev 69: 299-309.

10. Whyte JL, McArthur R, Topping D, et al. (1992) Oat bran lowers plasma cholesterol levels in mildly hypercholesterolemic men. J Am Diet Assoc 92: 446-449.

11. Smith RD, Kelly CN, Fielding BA, et al. (2003) Long-term monounsaturated fatty acid diets reduce platelet aggregation in healthy young subjects. Br J Nutr 90: 597-606.

12. Estruch R, Ros E, Salas-Salvadó J, et al. (2018) Primary prevention of cardiovascular disease with a mediterranean diet supplemented with extra-virgin olive oil or nuts. N Engl J Med 378: e34.

13. Nguyen T, Karl M, Santini A (2017) Red yeast rice. Foods 6: 19.

14. Heber D, Yip I, Ashley JM, et al. (1999) Cholesterol-lowering effects of a proprietary Chinese red-yeast-rice dietary supplement. Am J of Clin Nutr 69: 231-236.

15. Gerards MC, Terlou RJ, Yu H, et al. (2015) Traditional Chinese lipid-lowering agent red yeast rice re-sults in significant LDL-C reduction but safety is uncertain-A systematic review and metaanalysis. Atherosclerosis 240: 415-423.

16. Becker DJ, French B, Morris PB, et al. (2013) Phytosterols, red yeast rice, and lifestyle changes instead of statins: A randomized, double-blinded, placebo-controlled trial. Am Heart J 166: 187196.

17. Nakamura H, Arakawa K, Itakura H, et al. (2006) Primary prevention of cardiovascular disease with pravastatin in Japan (MEGA Study): A prospective randomised controlled trial. Lancet 368: 1155-1163.

18. Ridker PM, Danielson E, Fonseca FA, et al. (2008) Rosuvastatin to prevent vascular events in men and women with elevated C-reactive protein. N Engl J Med 359: 2195-2207.

19. Martinez-Villaluenga C, Penas E (2017) Health benefits of oat: Current evidence and molecular mechanisms. Current Opinion in Food Science 14: 26-31.

20. Violi F, Loffredo L, Pignatelli P, et al. (2015) Extra virgin olive oil use is associated with improved post-prandial blood glucose and LDL-C cholesterol in healthy subjects. Nutr Diabetes 5: e172. 
21. Joshi S, Mohan V (2018) Pros \& cons of some popular extreme weight-loss diets. Indian J Med Res 148: 642-647.
22. Hall KD, Kahan S (2018) Maintenance of lost weight and longterm management of obesity. Med Clin North Am 102: 183-197.

DOI: $10.36959 / 487 / 291$

Copyright: (C) 2021 Silva AO. This is an open-access article distributed under the terms of the Creative Commons Attribution License, which permits unrestricted use, distribution, and reproduction in any medium, provided the original author and source are credited. 